\title{
Comparative Analysis of the Digital Terrain Models Extracted from Airborne LiDAR Point Clouds Using Different Filtering Approaches in Residential Landscapes
}

\author{
Fahmy F. F. Asal (ㄷ) \\ Civil Engineering Department, Faculty of Engineering, Menoufia University, Shebin El-Kom, Egypt \\ Email: fahmy_asal@hotmail.com
}

How to cite this paper: Asal, F.F.F. (2019) Comparative Analysis of the Digital Terrain Models Extracted from Airborne LiDAR Point Clouds Using Different Filtering Approaches in Residential Landscapes. Advances in Remote Sensing, 8, 51-75. https://doi.org/10.4236/ars.2019.82004

Received: April 5, 2019

Accepted: June 25, 2019

Published: June 28, 2019

Copyright () 2019 by author(s) and Scientific Research Publishing Inc. This work is licensed under the Creative Commons Attribution International License (CC BY 4.0).

http://creativecommons.org/licenses/by/4.0/ (c) (i) Open Access

\begin{abstract}
Light Detection And Ranging (LiDAR) is a well-established active remote sensing technology that can provide accurate digital elevation measurements for the terrain and non-ground objects such as vegetations and buildings, etc. Non-ground objects need to be removed for creation of a Digital Terrain Model (DTM) which is a continuous surface representing only ground surface points. This study aimed at comparative analysis of three main filtering approaches for stripping off non-ground objects namely; Gaussian low pass filter, focal analysis mean filter and DTM slope-based filter of varying window sizes in creation of a reliable DTM from airborne LiDAR point clouds. A sample of LiDAR data provided by the ISPRS WG III/4 captured at Vaihingen in Germany over a pure residential area has been used in the analysis. Visual analysis has indicated that Gaussian low pass filter has given blurred DTMs of attenuated high-frequency objects and emphasized low-frequency objects while it has achieved improved removal of non-ground object at larger window sizes. Focal analysis mean filter has shown better removal of nonground objects compared to Gaussian low pass filter especially at large window sizes where details of non-ground objects almost have diminished in the DTMs from window sizes of $25 \times 25$ and greater. DTM slope-based filter has created bare earth models that have been full of gabs at the positions of the non-ground objects where the sizes and numbers of that gabs have increased with increasing the window sizes of filter. Those gaps have been closed through exploitation of the spline interpolation method in order to get continuous surface representing bare earth landscape. Comparative analysis has shown that the minimum elevations of the DTMs increase with increasing the filter widow sizes till $21 \times 21$ and $31 \times 31$ for the Gaussian low pass filter and
\end{abstract}


the focal analysis mean filter respectively. On the other hand, the DTM slope-based filter has kept the minimum elevation of the original data, that could be due to noise in the LiDAR data unchanged. Alternatively, the three approaches have produced DTMs of decreasing maximum elevation values and consequently decreasing ranges of elevations due to increases in the filter window sizes. Moreover, the standard deviations of the created DTMs from the three filters have decreased with increasing the filter window sizes however, the decreases have been continuous and steady in the cases of the Gaussian low pass filter and the focal analysis mean filters while in the case of the DTM slope-based filter the standard deviations of the created DTMs have decreased with high rates till window size of $31 \times 31$ then they have kept unchanged due to more increases in the filter window sizes.

\section{Keywords}

DSM/DEM/DTM, Airborne LiDAR Point Clouds, DSM Filtering, Gaussian

Low Pass Filter, Focal Analysis Mean Filter, DTM Slope-Based Filter, Removal of Non-Ground Objects

\section{Introduction}

Digital Terrain Model (DTM) of a specific area represents the ground surface elevations in that area. This is a very important surface model for modelling of the ground landscape in addition to the wide ranges of engineering and environmental applications that require a DTM. Also, DTM is crucial in planning of different types of infrastructure projects such as irrigation or sewerage systems. It is also, very important in the optimum design and building of road and transportation networks. These important applications and many others require the creation of an accurate DTM. Light Detection and Ranging (LiDAR) is a well-established active remote sensing technology that can provide accurate digital elevation measurements for the terrain and non-ground objects [1] [2] [3]. Also, it can collect measurements at wide ranges of resolutions that can suit wide ranges of applications through creation of high accurate and high-resolution Digital Surface Models (DSMs). It is also, important to note that the DSMs created from airborne LiDAR data are usually more detailed compared to those obtained from other types of data such as ground surveying or GPS measurements [4] [5]. Airborne LiDAR enjoys a wide range of applications such as mapping of corridors, rapid mapping, damage assessment following natural disasters, forest canopy height mapping and three-dimensional city modeling [6]. The most common process for such applications is extraction of DTMs through filtering of Airborne LiDAR measurements [7] since introduction of airborne LiDAR that also known airborne laser scanning has caused a revolution in topographic terrain capturing especially in extraction of DTM [8]. However, airborne LiDAR data usually contain measurements for non-ground objects such as ve- 
getation cover, buildings, etc., that need to be efficiently removed for creation of a reliable DTM representing the actual ground surface as if it is a bare earth surface [9]. The Extracted DTMs from airborne LiDAR measurements through stripping off non-ground objects can be employed in many applications such as mapping of the earth's surface and hydrodynamic modeling for flood risk assessments [1]. Thus, since the availability of airborne LiDAR measurements extraction of bare earth DTMs have become attainable with high accuracy through filtering of non-ground point from the high-density airborne LiDAR data termed as point cloud data [10]. Also, automated DTM extraction from remotely sensed data gained a wide range of areas of applications due to improvements in sensor resolution [11].

The majority of the approaches for extraction of DTMs from LiDAR measurements have been based on the concept of searching for the lowest points in a user-defined neighborhood by using morphological filters using curvature methods and identifying them as bare earth [4]. Different filtering algorithms have been developed to classify LiDAR point clouds into ground and non-ground points but challenges to identify complex objects such as bridges and eccentric roofs are still not overcome [6] [12]. Wang and Tseng, 2014 [10] state that, more recently, airborne LiDAR has been gradually replacing photogrammetry to become the chief method in extraction of DTMs as it has been a time and cost-effective method. This is motivated by the ability of a laser beam to penetrate a tree canopy making LiDAR a superior tool for DTM generation in forest areas. However, filtering of ground points is a crucial step for most applications of airborne LiDAR point clouds. Full-waveform LiDAR is an active technology of photogrammetry and remote sensing that can provide more detailed information about objects through laser pulse path compared to the discrete-return topographic LiDAR where point cloud and waveform information can be obtained by waveform decomposition [13].

Baligh et al. 2008 [14] carried out a research to determine the performance of three different filters and application of wavelets for minimizing noise in the LiDAR data. They acknowledged that the performances of the examined filters depend on the complexity of the landscapes while they found that wavelet denoising performed well. Zhang and Whitman 2005 [15] presented three methods for removing non-ground points from airborne LiDAR measurements including elevation threshold with expanding window, maximum local slope, and progressive morphological filters. They analyzed data points basing on variations of local slope and elevation in a test site of varied elevations and varied densities of trees, houses, and sand dunes. They acknowledged that the three filters can effectively remove most of the non-ground points in low-elevation urban and high elevation forested areas, however, the morphological filter achieved the best results in coastal barrier island areas, while the other filters tended to remove the tops of steep sand dunes. Chen et al. 2017 [16] reviewed the different methods for DTM generation from airborne LiDAR data. They acknowledged that extrac- 
tion of DTMs is the fundamental application of airborne laser scanning namely; airborne LiDAR, however, although big progress has been made in this objective, DTM generation from airborne LiDAR in specific terrain situations, remains a challenging issue. They introduced the general principles of DTM generation and reviewed DTM generation methods and classified them into six categories; 1) surface-based adjustment methods; 2) morphology-based filtering methods, 3) triangulated irregular network (TIN)-based refinement methods, 4) segmentation and classification methods, 5) statistical analysis methods and 6) multi-scale comparison methods. They recommend that DTM generation methods show similar difficulties when performed in sharply changing terrain, areas with high densities of non-ground objects and complicated landscapes however, they suggested that fusion of multi-sources of data with integration of different methods could be effective to overcome DTM extraction difficulties in different landscapes. Chang et al. 2008 [17] presented a strategy for automatic terrain extraction from LiDAR data based on the assumption that sudden elevation changes usually correspond to non-ground objects and cause relief displacements in perspective views where the introduced relief displacements occlude neighboring ground points. They generated a DSM through resampling irregular LiDAR point clouds to a regular grid, then by using synthesized projection centers located above the DSM and analyzing the visibility maps in perspective images the DSM can be classified into non-ground and ground hypotheses.

Classification of ground and non-ground points is an important issue for many applications of airborne LiDAR measurements. Hui et al. 2019 [18] presented a threshold-free filtering algorithm based on what they called expectation-maximization and on the assumption that point clouds are seen as a mixture of Gaussian models. They used mixed Gaussian model for partitioning of point clouds for separation of ground points and non-ground points from point clouds with the application of the expectation-maximization to realize the separation, which calculates the maximum likelihood estimates of the mixture parameters. Yunfei et al. 2008 [3] stated that extraction of accurate DTMs is one of important applications of airborne LiDAR so many different approaches have been developed to separate ground points from non-ground points, including mathematical morphology, adaptive and robust filtering, and unsupervised segmentation basing on geometric characteristics of LiDAR points. They presented an approach to separate vegetation points from ground points in a mountainous area mostly based on skewness change of LiDAR intensity information from both all laser returns and they recommended that such method can efficiently separate ground points from non-ground points in forested areas. Silva et al., 2018 [1] carried out a study that aimed to evaluate the performance of four ground filtering algorithms for DTM extraction from airborne LiDAR measurements in a forest environment of distinct classes of land use and land cover (e.g., shrubland, grassland, bare soil, and three forest types according to tree density and silvicultural interventions; closed-canopy forest, intermediate-canopy 
forest, and open-canopy forest), where the tested four ground filtering techniques are: weighted linear least squares, multi-scale curvature classification, progressive morphological filter and progressive triangulated irregular network. They recommend that the four algorithms performed well across the land cover but the progressive morphological filter yielded the least number of points classified as ground.

Sharma et al. 2010, [4] acknowledged that the topography and land cover determine infiltration, runoff, and erosion processes on watershed, however, time modeling and routing of surface water and erosion are determined by the digital elevation data that can be obtained from high-resolution ground-based LiDAR. They used a slope threshold and a focal mean filter method to remove vegetation and create bare earth DTMs and recommend that validations of the methods show vertical error of $\pm 7.5 \mathrm{~mm}$ in the final DTM. Sulaiman et al. 2010 [5] presented a study aimed to analyze the suitability of filtering method in Open-Source software to the extract DTM from airborne LiDAR measurements through a filtering process using Airborne LiDAR Data Processing and Analysis Tools (ALDPAT) which is an open source software. They used five different filters in ALDPAT to filter the same LiDAR data in addition to the use of TerraScan commercial software to process the same data. They evaluated the DTMs generated by ALDPAT through comparing them with the DTMs obtained from the TerraScan and recommended that Elevation Threshold with Expand Window filter has produced almost similar DTM as the one produced by TerraScan. Wang and Tseng, 2014 [10] state that Numerous automatic filters have been developed to remove non-ground data from airborne LiDAR measurements where a slope threshold is to be specified to classify points into ground and non-ground which lead to frequent over-filtering problems in cliff-like terrains. They proposed using a dual-directional slope-based filter originating from a conventional slope-based filter to overcome this problem where that filter was designed as a directional filter in one dimension and performed on every profile of LiDAR point where, a directional filter is first applied to the profile, and another directional filter is then applied at an angle of $180^{\circ}$ from the first one. The directional slope-based filters are complementing each other's for avoiding over-filtering. They recommend that the result showed that the proposed method gave a classification accuracy that was as good as most of the compared methods.

Rashidi and Rastiveis, 2018 [6] introduced an algorithm for ground filtering of LiDAR data based on the Slope and Progressive Window Thresholding (SPWT) through utilizing the slope between adjacent points and the elevation information of points in a local window to detect non-ground points. They evaluated the performance of the algorithm using low- and high-resolution datasets where they recommended that irrespective of data resolution, slope and progressive window thresholding filter could effectively remove non-ground points from airborne LiDAR data. Meng et al. 2010 [19] reviewed LiDAR ground filtering algorithms for creating DTMs with discussions of critical issues for implementa- 
tion of LiDAR ground filtering techniques such as filtering procedures for different landscapes, and site selection criteria, accuracy assessment and procedure classification. Their review highlighted three types of landscapes for which current ground filtering algorithms are suboptimal that can be refined in future studies including: surfaces with rough terrain or discontinuous slope, dense forest areas where laser pulses cannot penetrate in addition to regions with low vegetation that usually ignored by ground filters. Özcan et al. 2018 [11] presented a ground filtering and segmentation method that starts with extracting DSM feature points which are used to generate a non-ground object maps in a spatial domain to be used as seed points in a segmentation method based on morphological operations which lead to ground filtering and DTM generation. They recommended that their method enjoy robustness in handling urban areas of different properties with few parameters that need to be adjusted.

$\mathrm{Hu}$ et al. 2017 [20] exploited full-waveform LiDAR data to detect weak returns backscattered by the bare terrain underneath vegetation canopies for improvement of DTM generation. Also, they proposed an integrated approach where echo detection, terrain identification, and TIN generation were carried out iteratively. After testing the approach, they recommended that more terrain points under shrubs could be recognized and the generated DTMs provided more details in the terrain than those obtained using the progressive TIN method. Xing, et al. 2017 [13] proposed a surface fitting filtering method with waveform information through resolving discrete point clouds and waveform parameters followed by selecting ground seed points and detected by waveform parameters and robust estimation. Then, fitting terrain surface with height difference threshold determination in consideration of window size and mean square error. After that, the points ware classified gradually with rising of window size where the filtering process is not finished until window size is larger than the threshold. They stated that the accuracy of point cloud filtering was improved. Liu al. 2012 [21] presented a filtering algorithm based on grid partition using dynamic quad tress and moving least squares where points were partitioned and corresponding dynamic quad trees indices were established. Then, points in grids were employed to fit a DTM reference plane using moving least square technology. At the end, ground points were separated from non-ground ones if they were positioned above the reference plane and have a distance to the plane bigger than the threshold value. They recommended that the algorithm is of high precision and determined ground points effectively without losing detailed information of the terrain. Abdullah et al. 2012 [22] undertaken an assessment to examine the performance of seven different LiDAR filtering algorithms and to evaluate their suitability for urban flood modelling applications since they acknowledged that none of these algorithms can be regarded as fully suitable to support the job in its current form. They gave the augmentation of progressive morphological filtering algorithm for processing raw LiDAR data to be modified to incorporate buildings with basement, passage buildings and solid buildings. They recom- 
mended that differences in flood depths of $40 \%$ were noticed between a model basing on a DTM extracted by the progressive morphological filtering algorithm and the predictions of other models.

\section{Research Aims and Objectives}

This research aimed at exploration of the different approaches for filtering of Airborne LiDAR measurements for removal of non-ground objects in order to create a Digital Terrain Model (DTM) that can be employed in a wide range of engineering and environmental applications. In addition, it aimed at applying a comparative study of the application of three main filtering techniques namely; Gaussian low pass filter, focal analysis mean filter, and DTM slope-based filter on airborne LiDAR DSMs in urban residential landscape at varying filter window sizes for creation of a reliable DTM in addition to evaluation of the effects of the sizes of user-defined windows of Gaussian low pass filter, focal analysis mean filter, and DTM slope-based filter on the qualities the created DTMs.

\section{Test Site, Test Data and Methodology}

A sample of LiDAR data of the ISPRS WG III/4 Test Project on Urban Classification and 3D Building Reconstruction has been provided by ISPRS WG III/4 and the German Association of Photogrammetry and Remote Sensing (DGPF) [23]. The data set was captured over Vaihingen in Germany over a pure residential area with small detached houses on 21 August 2008 by Leica Geosystems using a Leica ALS50 system of $45^{\circ}$ field of view and average flying height of 500 $\mathrm{m}$ above ground. The whole airborne laser scanning data set of DGPF comprises 10 strips with average strip overlap of $30 \%$ and median point density of 6.7 points $/ \mathrm{m}^{2}$. However, point density varies considerably over the whole block depending on the overlap, but in the areas covered by only one strip, the mean point density is about 4.0 points $/ \mathrm{m}^{2}$ (Rottensteiner et al. 2013) [24]. In this study, the file Vaihingen_Strip_03.las has been used for creation and analysis of DTMs through filtering of airborne LiDAR DSM in urban residential landscape. As shown in Figure 1, Vaihingen _Strip_03.las test data file represents a complete Airborne laser scanning (ALS) strip and covers an area of about $725,000 \mathrm{~m}^{2}$ of average dimensions of about 500 meters in swath width by about 1450 meters as the swath length. The sample data consists of 3,774,279 LiDAR data measurements giving LiDAR point cloud density of 5.2059 points per one meter squared $\left(\mathrm{pts} / \mathrm{m}^{2}\right)$. This means that one LiDAR measurement has been recorded for every 0.1921 square meters in average. The statistical analysis of the data set has shown a minimum elevation of 165.203 meters and a maximum elevation of 298.932 meters giving a range of elevations of 133.729 meters, see Figure 1. Additionally, the mean elevation of the dataset is 265.441 meters and the standard deviation is 7.921 meters.

A Digital Surface Model (DSM), see Figure 2 has been created from 


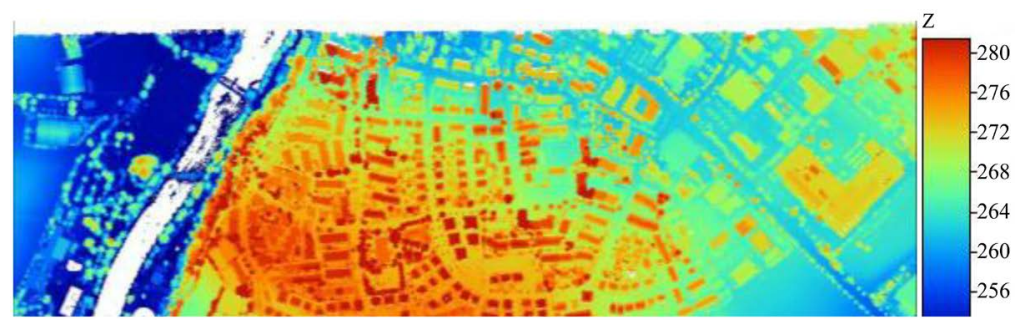

Figure 1. Point cloud airborne LiDAR dataset of the file Vaihingen_Strip_03.las

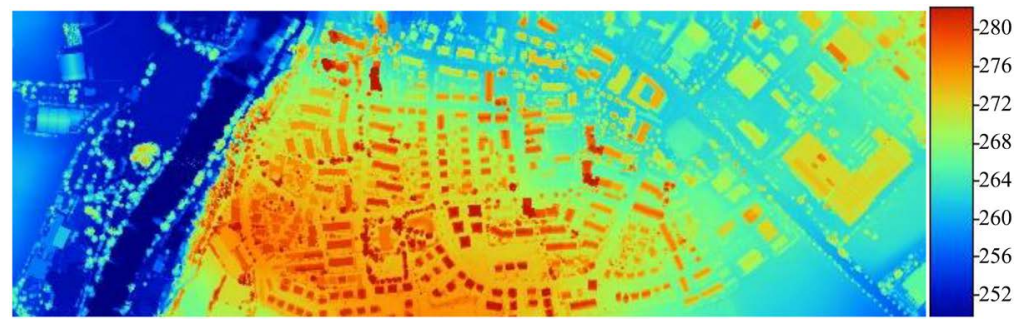

Figure 2. DSM created from point cloud airborne LiDAR dataset of the file Vaihingen_Strip_03.las

Vaihingen_Strip_03.las LiDAR data file using SAGA 6.4 open source GIS software where the Inverse Distance Weighting (IDW) interpolation method with power of four and grid resolution of half a meter has been used as the interpolation parameters. The generated DSM in Figure 2 has been subjected to filtering operations with different three filtering approaches, namely, the Gaussian low pass filtering approach, the focal analysis mean filtering method and the DTM slope-based filtering technique for the purpose of removal of non-ground objects and creation of Digital Terrain Models in the residential landscapes. The DTM filtering approach of the LiDAR DSM have been performed using different window sizes of $3 \times 3,5 \times 5,7 \times 7,9 \times 9,11 \times 11,15 \times 15,21 \times 21,25 \times 25,31 \times$ $31,35 \times 35$ and $41 \times 41$ under SAGA 6.4 open source GIS software and the Surfer 15 commercial software packages in order assess how the filter window size affects the removal of non-ground objects in creation of a reliable DTM. The generated DTMs from Gaussian low pass filter, focal analysis mean filter and DTM slope-based filter at varying filter window sizes have been subjected to visual and statistical analysis.

\section{Creation and Analysis of Digital Terrain Models with the Use of Gaussian Low Pass Filter}

Low pass filtering is a spatial filtering process based on using low pass filters designed to emphasize low spatial frequency features and deemphasize high spatial frequency features of an image in a spatial domain [25] [26]. Low-frequency information of an image represents the background patterns of the image. The output from low pass filtering of a digital image is an image of much of details in the original image are smoothed or removed. Thus, low pass filtering operation can be identified as a process of smoothing or blurring the digital image [27] 
[28]. Gaussian low pass filter is a smoothing filter used to blur or soften data and to remove details and noise from DSMs and raster images. The amount of smoothing is determined by the standard deviation where for higher standard deviations a larger search radius is required [18] [29] [30].

In this study Gaussian low pass filter has been performed on the LiDAR DSM, Figure 2 with varying window sizes for the purpose of removal of non-ground features and exploring how successful is the Gaussian low pass filter in creation of a reliable DTM. Also, assessment of the effects of the filter window size on the characteristics of the produced DTMs has been a main objective of the study. Figures 3-8 depict DTMs created from Gaussian low pass filtering of Airborne LiDAR DSM with window sizes of $3 \times 3,7 \times 7,15 \times 15,25 \times 25,35 \times 35$ and 41 $\times 41$ respectively. Compared to the DSM Figure 2, the DTM in Figure 3 is less detailed referring to removal of some noise and some of non-ground features as a result of applying Gaussian low pass filter with $3 \times 3$ window size. With increasing the window size of the filter to $7 \times 7$, no much differences can be observed between Figure 4 and Figure 3. A blurred DTM has been obtained in Figure 5 due to increasing the filter window size to $15 \times 15$ which is an indication of attenuation of some high frequencies representing non-ground objects with emphasizing of low frequencies pointing to the ground surface. With increasing the window size of Gaussian low pass filter much blurrier DTMs have been obtained as shown in Figures 6-8 depicting DTMs created with window sizes of $25 \times 25,35 \times 35$ and $41 \times 41$ respectively which refer to better removal of non-ground objects.

Table 1 depicts the statistical analysis of the DTMs created from filtering of LiDAR DSM with Gaussian low pass filter of varying window sizes of $3 \times 3,7 \times$ $7,15 \times 15,21 \times 21,25 \times 25,31 \times 31,35 \times 35$ and $41 \times 41$ in addition to the statistical analysis results of the airborne LiDAR DSM. From Table 1 it can be noticed that the minimum elevations, maximum elevations, ranges of elevation, standard deviations of elevation in addition to skewness and kurtosis of the DTMs have decreased with increasing the window size of Gaussian low pass filter due to attenuation of the high frequencies and emphasizing of the low frequencies. In the meantime, the mean, median and root mean square of the DTMs have not been affected much due to application of the Gaussian low pass filter of varying window sizes.

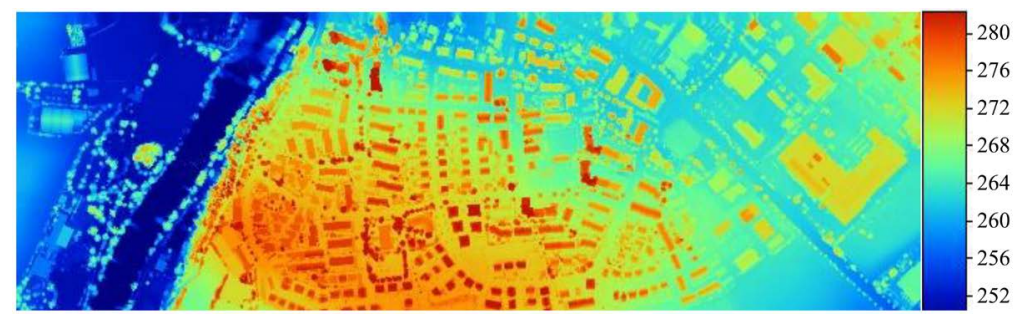

Figure 3. DTM created from low pass Gaussian filtering of Airborne LiDAR DSM with window size of $3 \times 3$. 


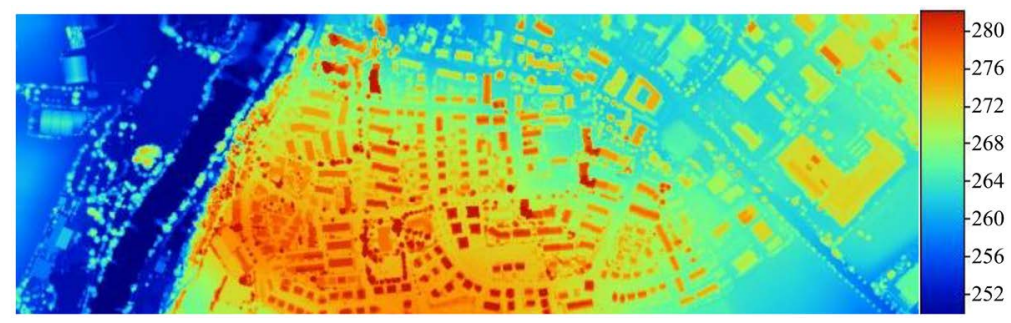

Figure 4. DTM created low pass from Gaussian filtering of Airborne LiDAR DSM with window size of $7 \times 7$.

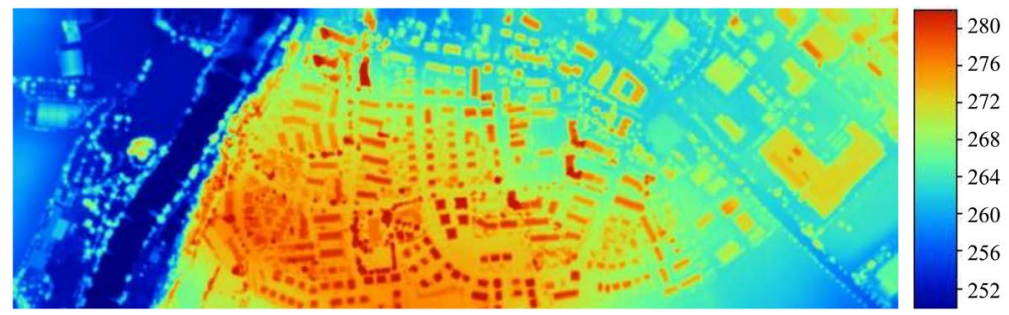

Figure 5. DTM created from low pass Gaussian filtering of Airborne LiDAR DSM with window size of $15 \times 15$.

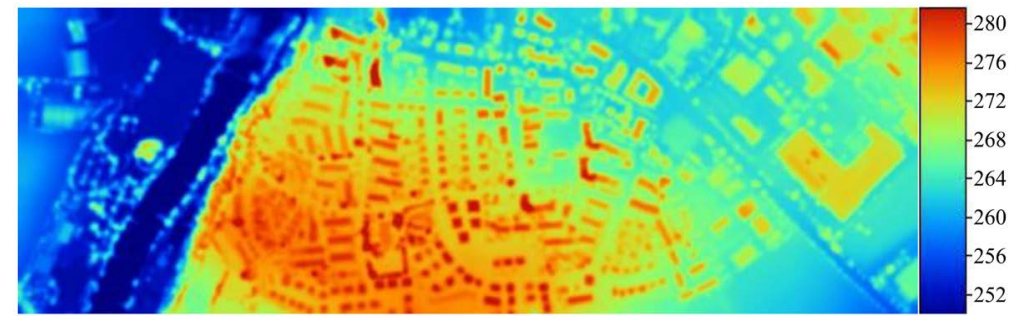

Figure 6. DTM created from low pass Gaussian filtering of Airborne LiDAR DSM with window size of $25 \times 25$.

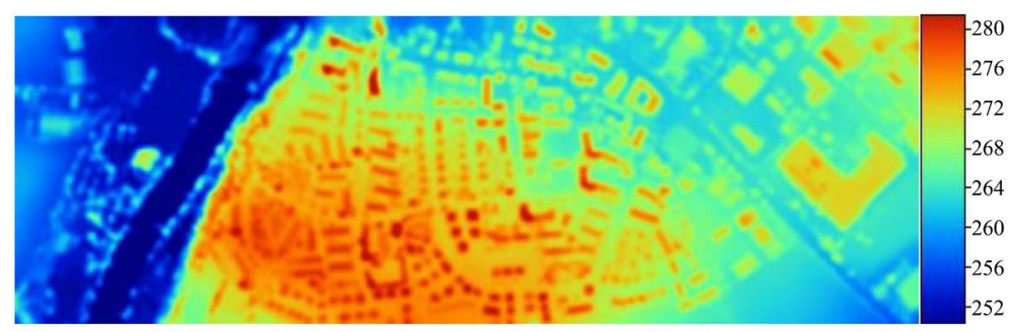

Figure 7. DTM created from low pass Gaussian filtering of Airborne LiDAR DSM with window size of $35 \times 35$.

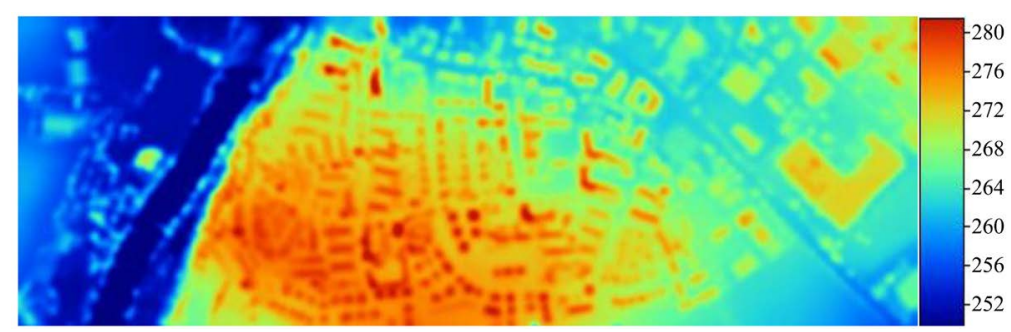

Figure 8. DTM created fromlow pass Gaussian filtering of Airborne LiDAR DSM with window size of $41 \times 41$. 
Table 1. Statistical analysis of the DTMs created from filtering of LiDAR DSM with Gaussian low pass filter of varying window sizes.

\begin{tabular}{|c|c|c|c|c|c|c|c|c|c|}
\hline $\begin{array}{c}\begin{array}{c}\text { Gaussian low } \\
\text { pass filter } \\
\text { window size }\end{array} \\
\text { Statistical } \\
\text { quantity }\end{array}$ & $\begin{array}{c}\text { Airborne } \\
\text { LiDAR } \\
\text { DSM }\end{array}$ & $\begin{array}{l}\text { DTM from } \\
\text { filter } 3 \times 3\end{array}$ & $\begin{array}{l}\text { DTM from } \\
\text { filter } 7 \times 7\end{array}$ & $\begin{array}{l}\text { DTM from } \\
\text { filter } 15 \times 15\end{array}$ & $\begin{array}{l}\text { DTM from } \\
\text { filter } 21 \times 21\end{array}$ & $\begin{array}{l}\text { DTM from } \\
\text { filter } 25 \times 25\end{array}$ & $\begin{array}{l}\text { DTM from } \\
\text { filter } 31 \times 31\end{array}$ & $\begin{array}{l}\text { DTM from } \\
\text { filter } 35 \times 35\end{array}$ & $\begin{array}{l}\text { DTM from } \\
\text { filter } 41 \times 41\end{array}$ \\
\hline Min. (m) & 165.259 & 165.858 & 182.825 & 223.183 & 236.151 & 242.958 & 245.415 & 245.517 & 245.644 \\
\hline Max. (m) & 292.764 & 291.445 & 289.265 & 289.079 & 287.991 & 286.884 & 285.822 & 284.944 & 283.984 \\
\hline Range (m) & 127.504 & 125.588 & 106.440 & 65.896 & 51.840 & 43.926 & 40.408 & 39.426 & 38.339 \\
\hline Mean $(\mathrm{m})$ & 265.289 & 265.289 & 265.289 & 265.289 & 265.289 & 265.289 & 265.290 & 265.290 & 265.290 \\
\hline Median (m) & 265.381 & 265.393 & 265.455 & 265.550 & 265.606 & 265.654 & 265.689 & 265.710 & 265.746 \\
\hline $\begin{array}{l}\text { Root Mean } \\
\text { Square (m) }\end{array}$ & 265.425 & 265.425 & 265.423 & 265.419 & 265.417 & 265.415 & 265.413 & 265.412 & 265.411 \\
\hline $\begin{array}{c}\text { Standard } \\
\text { Deviation }(\mathrm{m})\end{array}$ & 8.487 & 8.468 & 8.406 & 8.297 & 8.223 & 8.156 & 8.097 & 8.062 & 8.015 \\
\hline Skewness (m) & -0.318 & -0.321 & -0.332 & -0.354 & -0.372 & -0.387 & -0.401 & -0.408 & -0.417 \\
\hline Kurtosis (m) & 2.595 & 2.585 & 2.524 & 2.464 & 2.444 & 2.428 & 2.414 & 2.405 & 2.394 \\
\hline
\end{tabular}

\section{Creation and Analysis of Digital Terrain Models with the Use of Focal Analysis Mean Filter}

Focal analysis mean filter has been acknowledged by some authors as a method for smoothing of the DSMs and attenuation of the high frequencies [4] [9]. In this study focal analysis mean filter is performed on airborne LiDAR DSM, Figure 2 with varying window sizes for the purpose of removal of non-ground features and assessment of how successful is the focal analysis mean filter in creation of a reliable DTM in addition to uncover the effects of the filter window size on the characteristics of the produced DTMs. Figures 9-17 depict DTMs created from focal analysis mean filtering of Airborne LiDAR DSM, Figure 1 with window sizes of $3 \times 3,7 \times 7,15 \times 15,25 \times 25,35 \times 35$ and $41 \times 41$ respectively. Compared to the DSM in Figure 2, the DTM in Figure 9 does not show much differences which means that a focal analysis mean filter of $3 \times 3$ has not left much changes on the visual analysis of the created DTM. With increasing the window size to $7 \times 7$ a bit blurred DTM, see Figure 10 has been obtained compared to Figure 9. However, a blurred DTM has been obtained in Figure 11 due to increasing the filter window size to $15 \times 15$ which is an indication of removal of considerable numbers of non-ground objects. With increasing the window sizes of the focal analysis mean filter more than $15 \times 15$ much-blurred DTMs have been obtained as shown in Figures 12-14 which depict DTMs that have been obtained with the use of focal analysis mean filter of window sizes of $25 \times$ $25,35 \times 35$ and $41 \times 41$ respectively. This refers to better removal of most of non-ground objects. 


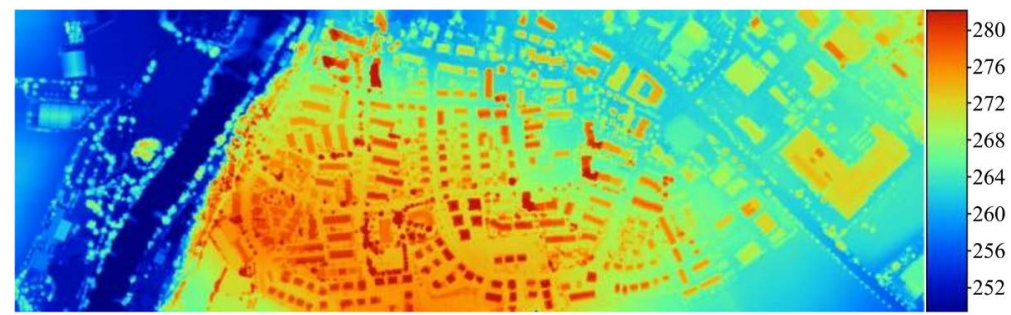

Figure 9. DTM created from focal analysis mean filtering of Airborne LiDAR DSM with window size of $3 \times 3$.

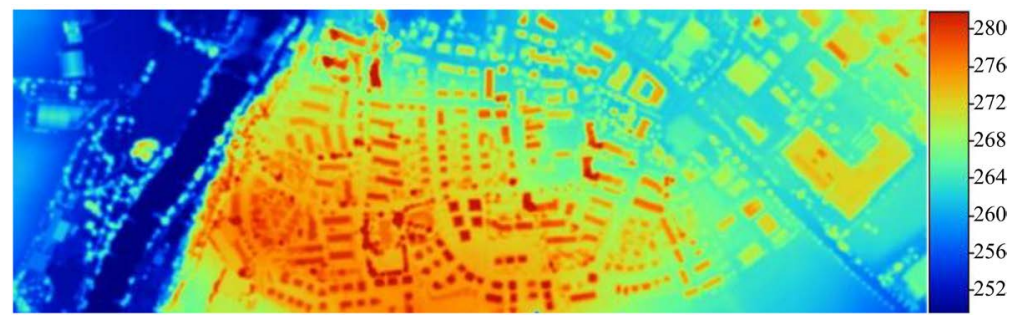

Figure 10. DTM created from focal analysis mean filtering of Airborne LiDAR DSM with window size of $7 \times 7$.

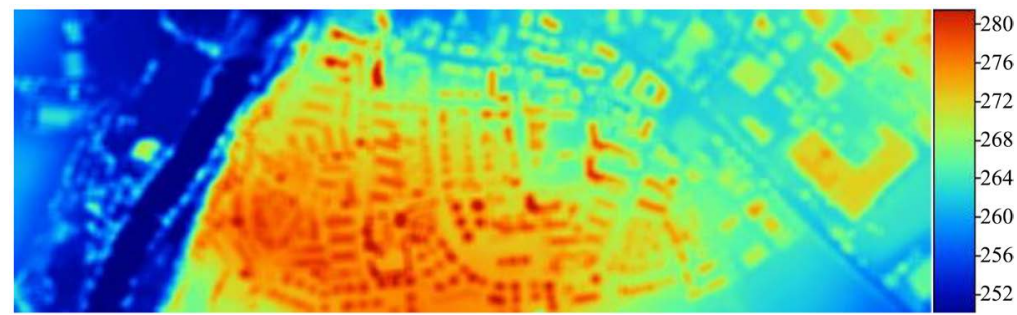

Figure 11. DTM created from focal analysis mean filtering of Airborne LiDAR DSM with window size of $15 \times 15$.

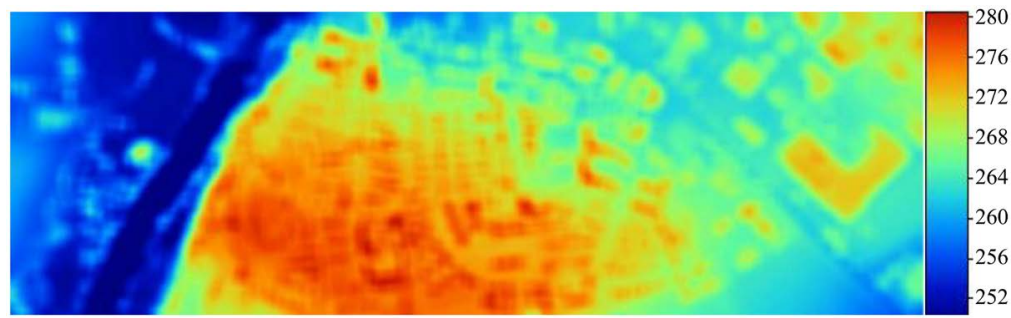

Figure 12. DTM created from focal analysis mean filtering of Airborne LiDAR DSM with window size of $25 \times 25$.

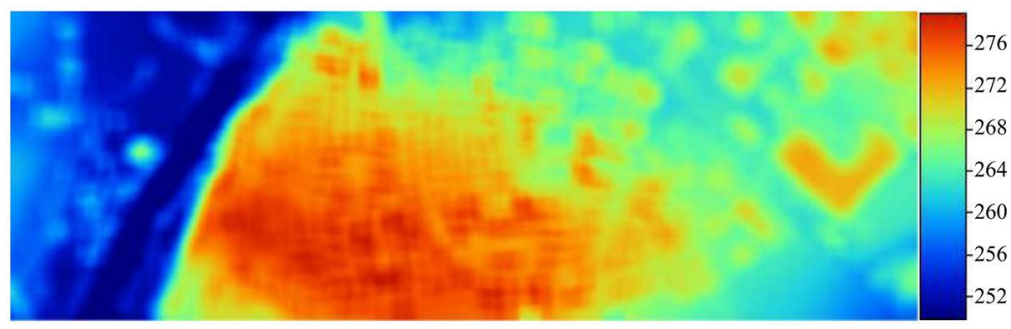

Figure 13. DTM created from focal analysis mean filtering of Airborne LiDAR DSM with window size of $35 \times 35$. 


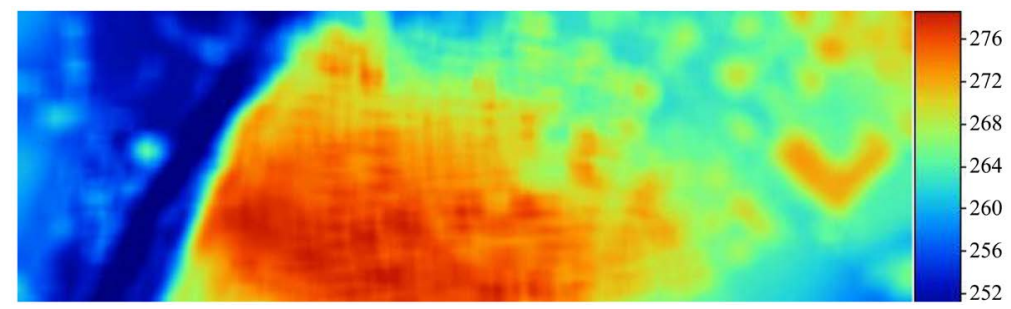

Figure 14. DTM created from focal analysis mean filtering of Airborne LiDAR DSM with window size of $41 \times 41$.

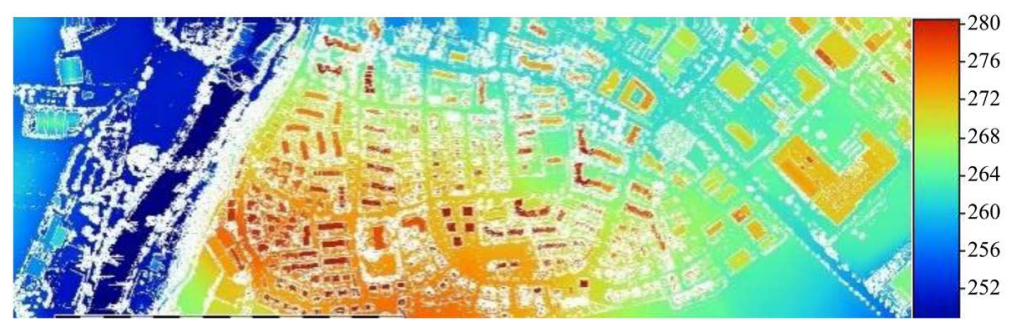

Figure 15. Bare earth modelextracted from DTM slope-based filtering of Airborne LiDAR DSM with window size of $3 \times 3$.

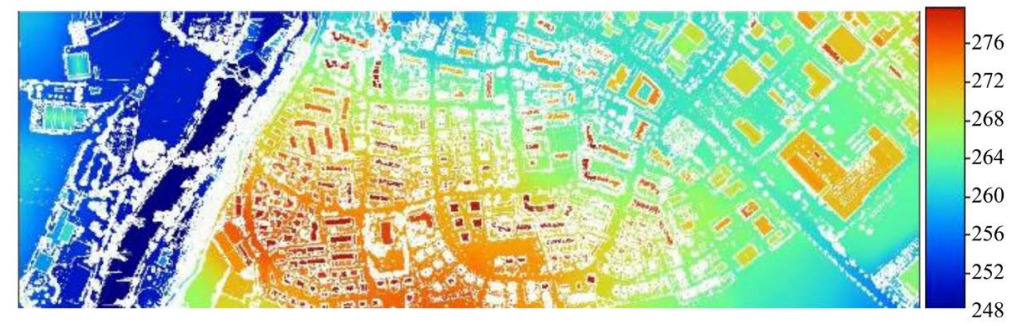

Figure 16. Bare earth modelextracted from DTM slope-based filtering of Airborne LiDAR DSM with window size of $7 \times 7$.

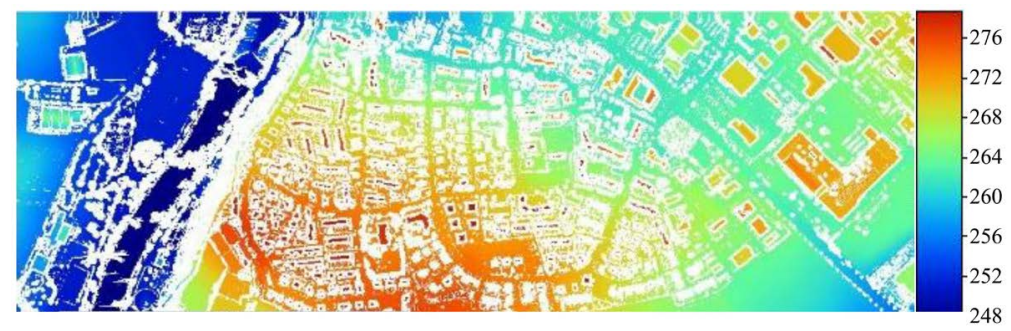

Figure 17. Bare earth modelextracted from DTM slope-based filtering of Airborne LiDAR DSM with window size of $15 \times 15$.

Table 2 depicts the statistical analysis results of the DTMs created from filtering of LiDAR DSM with focal analysis mean filter of varying window sizes of $3 \times 3,7 \times 7,15 \times 15,21 \times 21,25 \times 25,31 \times 31,35 \times 35$ and $41 \times 41$ respectively in addition to the statistical analysis results of the airborne LiDAR DSM. Similar to the case of DTM creation from Gaussian low pass filter and from Table 2, it can be noticed that the minimum elevations, maximum elevations, ranges of elevations, standard deviations of elevations in addition to skewness and kurtosis of the DTMs have decreased with increasing the window sizes of the focal analy- 
sis mean filter. However, the mean, median and root mean square of the created DTMs have not been affected much with the use of focal analysis mean filter of varying window sizes.

\section{Creation and Analysis of Digital Terrain Models with the Use of DTM Slope-Based Filter}

DTM slope-based filter is a grid filtering approach that works under the open source GIS software, SAGA (System for Automated Scientific Analysis) and can be used to filter a DSM to classify the grid cells into a bare earth layer and a removed object layer that also known as ground layer and non-ground layer respectively. The filtering approach is based on the concepts described by Vosselman, 2000 [31] assuming that a big elevation difference between two neighboring grid cells is unlikely to be caused by a steep slope in the terrain (Wichmann, 2010, Vosselman, 2000) [30] [32]. Thus, the probability that the higher elevation value grid cell could be a ground point decreases if the distance between the two grid cells decreases. In addition, the filter defines the acceptable height difference between the two grid cells as a function of the distance between them. Then, the grid cell is classified as ground if there is no other grid cell within the filter search radius such that the height difference between these grid cells is larger than the allowed maximum height difference at the distance between these grid cells. Moreover, an approximate ground surface slope variable is used to modify the filter function to match the overall slope in the whole DSM area. Furthermore, a confidence interval could be applied for omission of blunders [32] [33] [34].

Table 2. Statistical analysis of the DTMs created from filtering of LiDAR DSM with focal analysis mean filter of varying window sizes.

\begin{tabular}{|c|c|c|c|c|c|c|c|c|c|}
\hline $\begin{array}{c}\begin{array}{c}\text { Focal mean } \\
\text { filter window } \\
\text { size }\end{array} \\
\begin{array}{c}\text { Statistical } \\
\text { quantity }\end{array}\end{array}$ & $\begin{array}{c}\text { Airborne } \\
\text { LiDAR DSM }\end{array}$ & $\begin{array}{l}\text { DTM from } \\
\text { filter } 3 \times 3\end{array}$ & $\begin{array}{l}\text { DTM from } \\
\text { filter } 7 \times 7\end{array}$ & $\begin{array}{l}\text { DTM from } \\
\text { filter } 15 \times 15\end{array}$ & $\begin{array}{l}\text { DTM from } \\
\text { filter } 21 \times 21\end{array}$ & $\begin{array}{c}\text { DTM from } \\
\text { filter } 25 \times 25\end{array}$ & $\begin{array}{l}\text { DTM from } \\
\text { filter } 31 \times 31\end{array}$ & $\begin{array}{l}\text { DTM from } \\
\text { filter } 35 \times 35\end{array}$ & $\begin{array}{l}\text { DTM from } \\
\text { filter } 41 \times 41\end{array}$ \\
\hline Min. (m) & 165.259 & 168.355 & 200.914 & 239.984 & 245.254 & 245.435 & 245.553 & 245.636 & 245.750 \\
\hline Max. (m) & 292.764 & 290.246 & 289.328 & 288.654 & 286.975 & 286.516 & 284.712 & 283.665 & 282.209 \\
\hline Range (m) & 127.504 & 121.890 & 88.414 & 48.670 & 41.720 & 41.081 & 39.159 & 38.028 & 36.459 \\
\hline Mean (m) & 265.289 & 265.289 & 265.289 & 265.289 & 265.290 & 265.290 & 265.290 & 265.289 & 265.289 \\
\hline Median (m) & 265.381 & 265.417 & 265.495 & 265.605 & 265.667 & 265.698 & 265.744 & 265.768 & 265.801 \\
\hline $\begin{array}{l}\text { Root Mean } \\
\text { Square (m) }\end{array}$ & 265.425 & 265.424 & 265.421 & 265.417 & 265.414 & 265.413 & 265.411 & 265.410 & 265.408 \\
\hline $\begin{array}{c}\text { Standard } \\
\text { Deviation }(\mathrm{m})\end{array}$ & 8.487 & 8.441 & 8.368 & 8.228 & 8.140 & 8.089 & 8.023 & 7.986 & 7.937 \\
\hline Skewness (m) & -0.318 & -0.326 & -0.339 & -0.370 & -0.391 & -0.403 & -0.416 & -0.423 & -0.430 \\
\hline Kurtosis (m) & 2.595 & 2.561 & 2.493 & 2.445 & 2.425 & 2.413 & 2.397 & 2.388 & 2.377 \\
\hline
\end{tabular}


Figures 15-20 depict bare earth models extracted from DTM slope-based filtering of airborne LiDAR DSM with window sizes of $3 \times 3,7 \times 7,15 \times 15,25 \times$ $25,35 \times 35$ and $41 \times 41$ respectively. As shown in the figures the DTM slope-based filter has removed non-ground object leaving bare earth models that can be DTMs of the test area but with clear gaps of no data at the positions of the removed non-ground objects. In Figure 15 which is a bare earth model from the DTM slope-based filter of window size of $3 \times 3$ the filter has removed some of the non-ground objects but many of them are still there in the bare earth model. The numbers and sizes of the removed objects have increased with increasing the window size of the DTM slope-based filter to $7 \times 7$ as shown Figure 16 , where increasing of numbers of bigger white color patches at the positions of the non-ground objects can be interpretable in the bare earth model. Also, it can be interpretable that the ground areas are represented with same color tones in the Figures 15-20. Also, the DTM slope-based filter has achieved better removal of the non-ground objects at larger window sizes which is clear in Figures 18-20 which represent bare earth models obtained from the application of the DTM slope-based filter with window sizes of $25 \times 25,35 \times 35$ and $41 \times 41$ respectively on the LiDAR DSM.

Table 3 shows the statistical analysis of results of the bare earth models obtained from DTM slope-based filtering of the airborne LiDAR DSM, Figure 2 with varying window sizes of $3 \times 3,7 \times 7,15 \times 15,21 \times 21,25 \times 25,31 \times 31,35 \times$ 35 and $41 \times 41$ respectively in addition to the statistical analysis results of the airborne LiDAR DSM. Form Table 3 and different from the cases of DTM

Table 3. Statistical analysis results of the bare earth models created from filtering of airborne LiDAR DSM with DTM slope-based filter of varying window sizes.

\begin{tabular}{|c|c|c|c|c|c|c|c|c|c|}
\hline $\begin{array}{c}\text { Slope based } \\
\text { filter window } \\
\text { size }\end{array}$ & \multirow{2}{*}{$\begin{array}{c}\text { Airborne } \\
\text { LiDAR } \\
\text { DSM }\end{array}$} & \multirow[t]{2}{*}{$\begin{array}{l}\text { DTM from } \\
\text { filter } 3 \times 3\end{array}$} & \multirow[t]{2}{*}{$\begin{array}{l}\text { DTM from } \\
\text { filter } 7 \times 7\end{array}$} & \multirow[t]{2}{*}{$\begin{array}{l}\text { DTM from } \\
\text { filter } 15 \times 15\end{array}$} & \multirow[t]{2}{*}{$\begin{array}{l}\text { DTM from } \\
\text { filter } 21 \times 21\end{array}$} & \multirow[t]{2}{*}{$\begin{array}{l}\text { DTM from } \\
\text { filter } 25 \times 25\end{array}$} & \multirow[t]{2}{*}{$\begin{array}{l}\text { DTM from } \\
\text { filter } 31 \times 31\end{array}$} & \multirow[t]{2}{*}{$\begin{array}{l}\text { DTM from } \\
\text { filter } 35 \times 35\end{array}$} & \multirow[t]{2}{*}{$\begin{array}{l}\text { DTM from } \\
\text { filter } 41 \times 41\end{array}$} \\
\hline $\begin{array}{l}\text { Statistical } \\
\text { quantity }\end{array}$ & & & & & & & & & \\
\hline Min. (m) & 165.259 & 165.259 & 165.259 & 165.259 & 165.259 & 165.259 & 165.259 & 165.259 & 165.259 \\
\hline Max. (m) & 292.764 & 289.281 & 289.252 & 286.208 & 286.179 & 285.617 & 285.538 & 280.444 & 280.444 \\
\hline Range (m) & 127.504 & 124.022 & 123.993 & 120.949 & 120.920 & 120.358 & 120.279 & 115.185 & 115.185 \\
\hline Mean (m) & 265.289 & 263.873 & 263.467 & 262.912 & 262.677 & 262.590 & 262.530 & 262.502 & 262.470 \\
\hline Median (m) & 265.381 & 263.947 & 263.654 & 263.263 & 263.137 & 263.088 & 263.049 & 263.029 & 263.008 \\
\hline $\begin{array}{l}\text { Root Mean } \\
\text { Square }(m)\end{array}$ & 265.425 & 264.002 & 263.592 & 263.030 & 262.792 & 262.703 & 262.643 & 262.615 & 262.583 \\
\hline $\begin{array}{c}\text { Standard } \\
\text { Deviation }(\mathrm{m})\end{array}$ & 8.487 & 8.265 & 8.116 & 7.872 & 7.762 & 7.727 & 7.714 & 7.710 & 7.707 \\
\hline Skewness (m) & -0.318 & -0.267 & -0.269 & -0.295 & -0.313 & -0.315 & -0.307 & -0.301 & -0.293 \\
\hline Kurtosis (m) & 2.595 & 2.443 & 2.436 & 2.403 & 2.371 & 2.357 & 2.350 & 2.348 & 2.347 \\
\hline
\end{tabular}




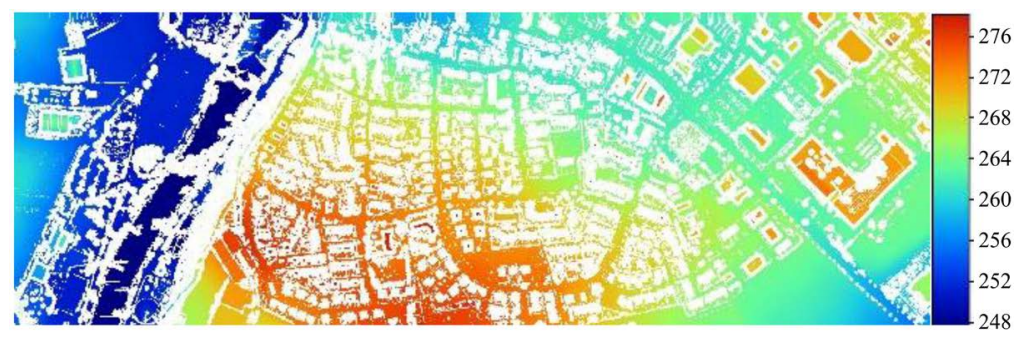

Figure 18. Bare earth modelextracted from DTM slope-based filtering of Airborne LiDAR DSM with window size of $25 \times 25$.

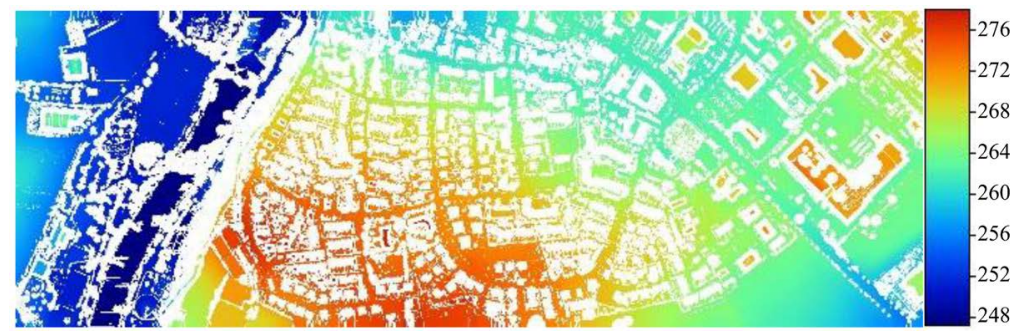

Figure 19. Bare earth modelextracted from DTM slope-based filtering of Airborne LiDAR DSM with window size of $35 \times 35$.

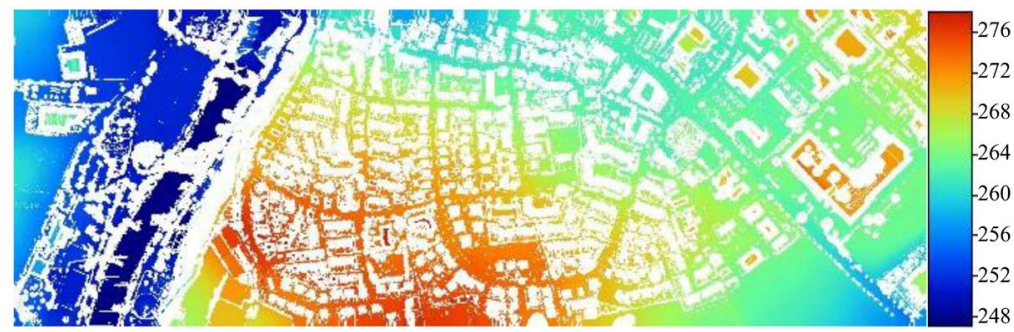

Figure 20. Bare earth modelextracted from DTM slope-based filtering of Airborne LiDAR DSM with window size of $41 \times 41$.

extraction from Gaussian low pass filter and focal analysis mean filter the minimum elevations of the created bare earth models have kept unchanged with all the applied window sizes of the DTM slope-based filter. This means that the DTM slope-based filter keeps the minimum elevation of the original DSM unchanged. On the other hand, the maximum elevations, ranges of elevations, means of elevations, medians of elevations, root mean squares of elevations, the standard deviations of elevations and kurtosis of the created bare earth models decreased with increasing the window sizes of the DTM slope-based filter. However, the skewness of the created bare earth models has increased with increasing the window size of the DTM slope-based filter.

As shown in Figures 15-20 the created bare earth models from the application of the DTM slope-based filter of varying window sizes are full of no data gaps at the positions of the removed objects that affect their exploitation as DTMs in various applications. So, these bare earth models need to be subjected to an interpolation technique to fill the gaps and obtain a continuous surface representing the earth's surface as a DTM. In this study, the created bare earth 
models, Figures 15-20 have been subjected the tool "close the gaps with spline" under SAGA-GIS software package to fill all gaps in the bare earth models and obtain continuous DTMs as depicted in Figures 21-26. In Figure 21 which is a DTM after filling the gap of the bare earth model from $3 \times 3$ it can be noticed that small numbers of non-ground objects have been removed, however, increasing numbers of removed non-ground objects can be observed in Figure 22 which is a closed gab DTM from $7 \times 7$ DTM slope-based filter but many non-ground objects yet have not been removed.

Better removal of non-ground objects can be observed in Figure 23 which is a closed gab DTM from $15 \times 15$ DTM slope-based filter but considerable numbers of non-ground objects yet have not been removed. However, increased removal of non-ground objects has been achieved with filter size of $25 \times 25$ but fewer numbers of sparse non-ground objects are still there, see Figure 24 . With increasing the window sizes of the DTM slope-based filter to $35 \times 35$ and $41 \times 41$ it can be interpretable that most of the non-ground objects have been removed as depicted in Figure 25 and Figure 26 respectively of views that can be close to a real DTM of a topographical bare earth surface.

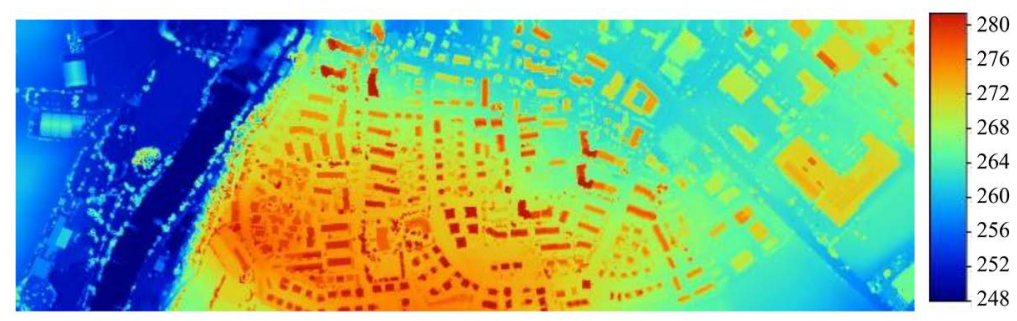

Figure 21. DTM created from DTM slope-based filtering of Airborne LiDAR DSM with window size of $3 \times 3$ after closing the gaps.

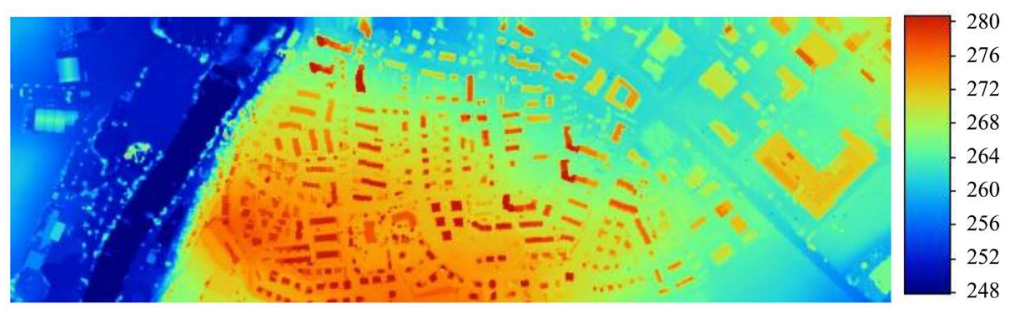

Figure 22. DTM created from DTM slope-based filtering of Airborne LiDAR DSM with window size of $7 \times 7$ after closing the gaps.

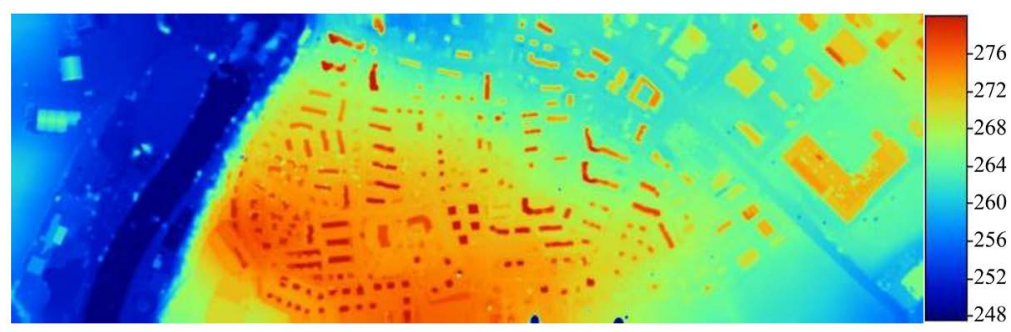

Figure 23. DTM created from DTM slope-based filtering of Airborne LiDAR DSM with window size of $15 \times 15$ after closing the gaps. 


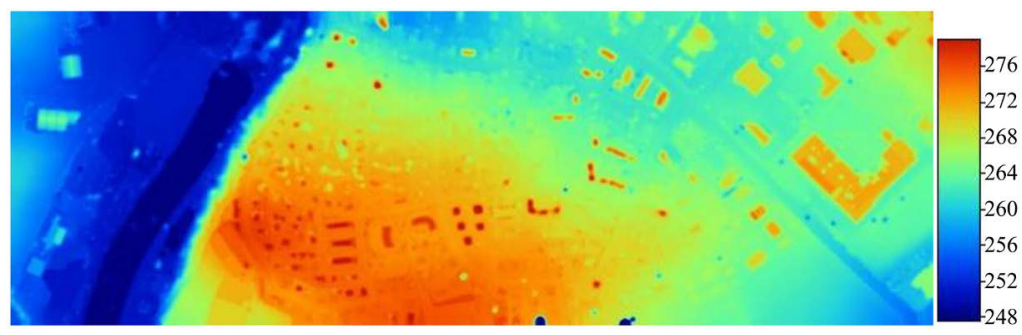

Figure 24. DTM created from DTM slope-based filtering of Airborne LiDAR DSM with window size of $25 \times 25$ after closing the gaps.

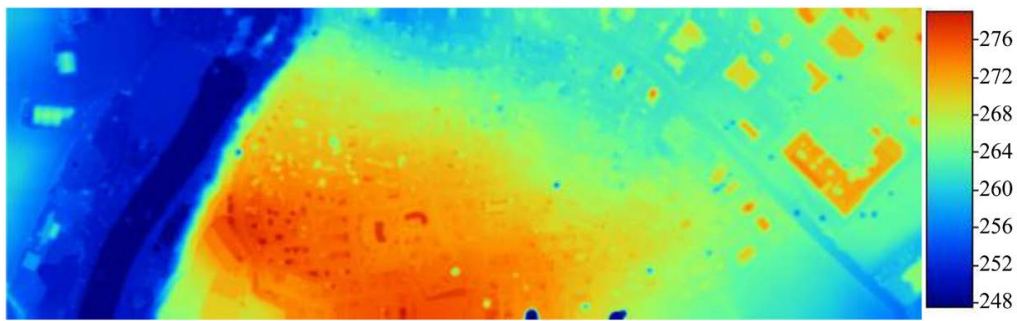

Figure 25. DTM created from DTM slope-based filtering of Airborne LiDAR DSM with window size of $35 \times 35$ after closing the gaps.

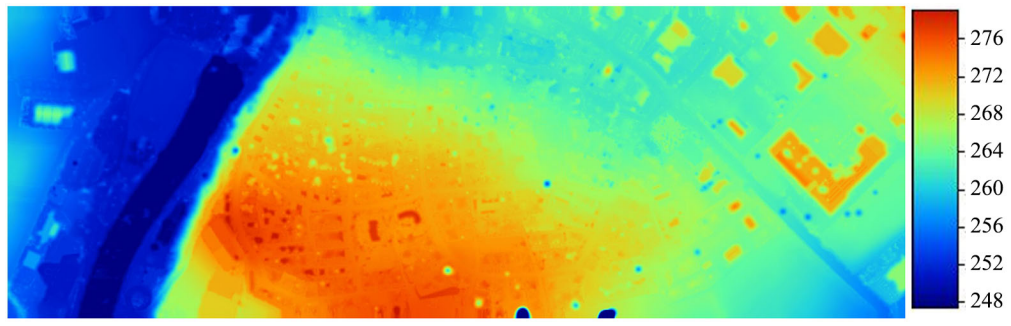

Figure 26. DTM created from DTM slope-based filtering of Airborne LiDAR DSM with window size of $41 \times 41$ after closing the gaps.

\section{Comparative Analysis of the Results}

Figures 27-32 depict charts that show comparative analysis of the DTMs produced from the three filters; the Gaussian low pass filter, the focal analysis mean filter and the DTM slope-based filter. In Figure 27 the minimum elevations of the DTMs from the Gaussian low pass filter and those from the focal analysis mean filter have increased with increasing the widow sizes of the filters which has not been the case with bare earth models produced from the DTM slope-based filter since this filtering approach has kept the minimum elevation of the original DSM unchanged despite increasing the window sizes of the filter. Also, from the chart Figure 27, the minimum elevations of the created DTM from the Gaussian low pass filter has kept unchanged with window sizes bigger than $21 \times 21$ while it has kept unchanged with window sizes bigger than $31 \times 31$ in the case of the focal analysis mean filter. This can be observed as the two curves of the Gaussian low pass filter and the focal analysis mean filter coincide together with same values of the minimum elevations of the produced DTMs from both filtering approaches. This has not been the case in Figure 28 which 
depict the maximum elevations of the produced DTMs against the window sizes of the examined three filtering approaches. The three filtering approaches have produced DTMs of maximum elevations that have decreased due to increases in

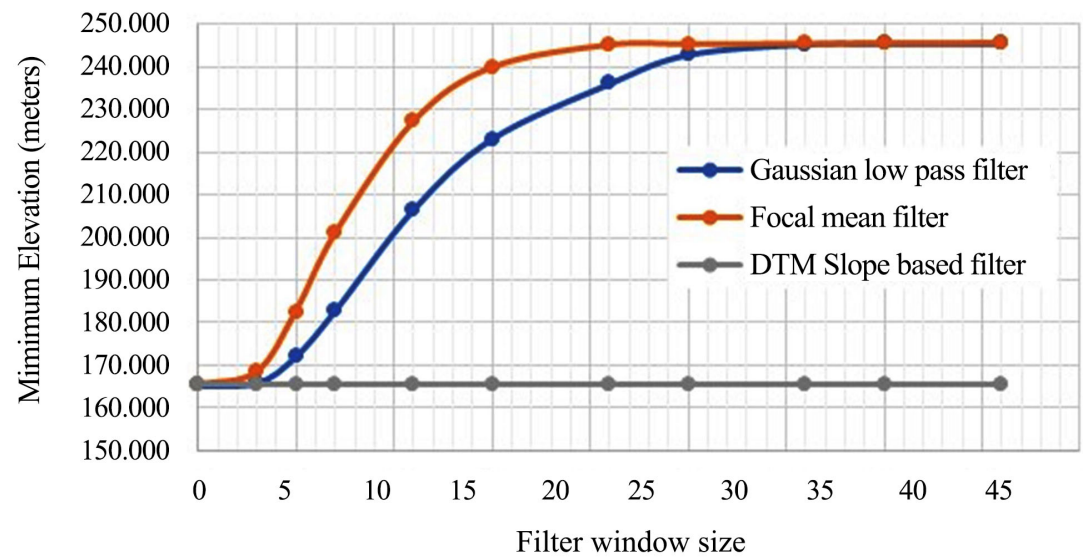

Figure 27. The relationship between the minimum elevations of the created DTMs from different filtering techniques and the filter window size.

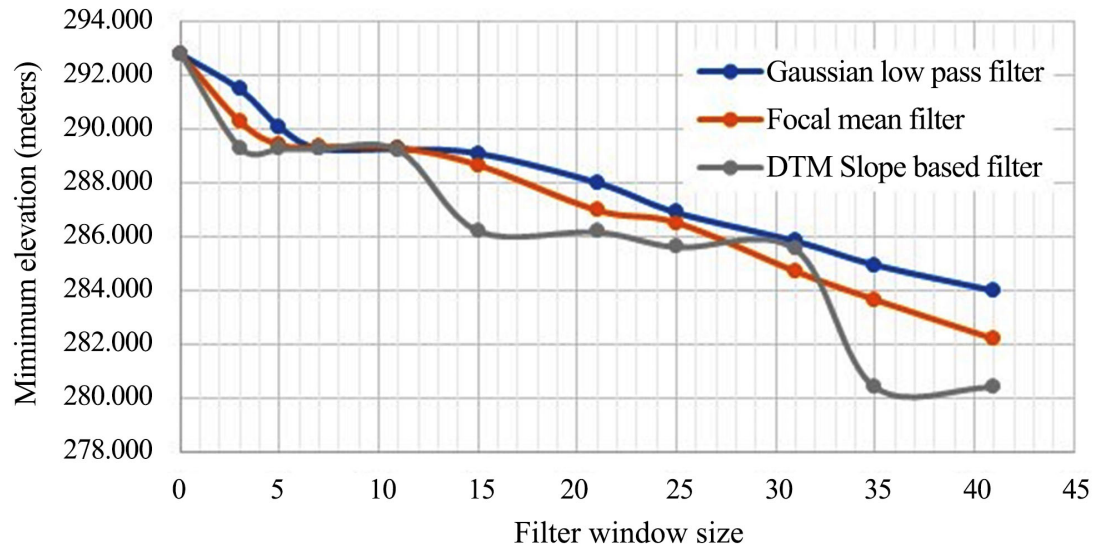

Figure 28. The relationship between the maximum elevations of the created DTMs from different filtering techniques and the filter window size.

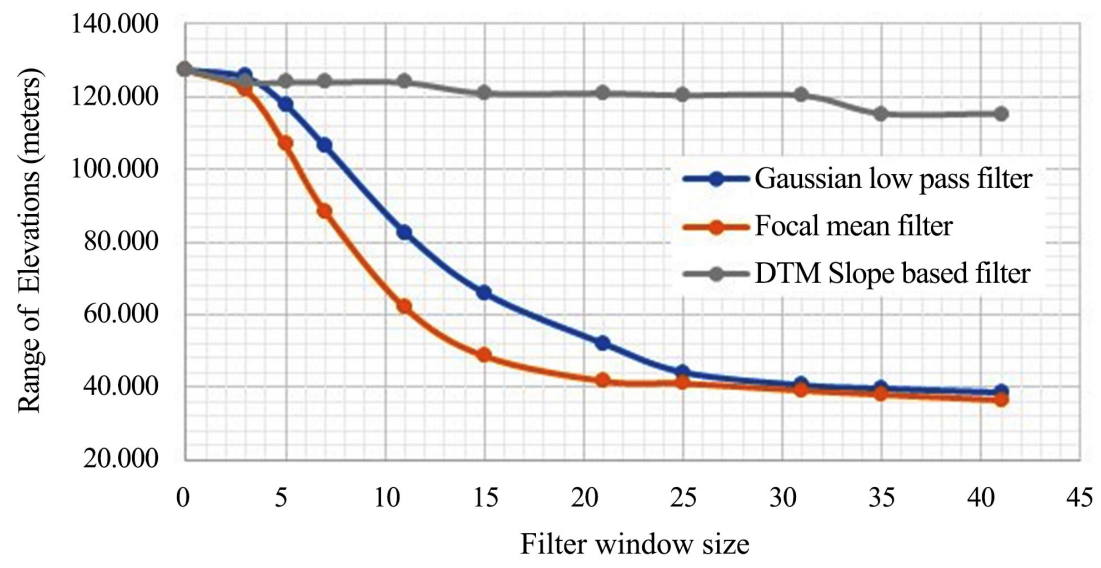

Figure 29. The relationship between the ranges of elevations of the created DTMs from different filtering techniques and the filter window size. 


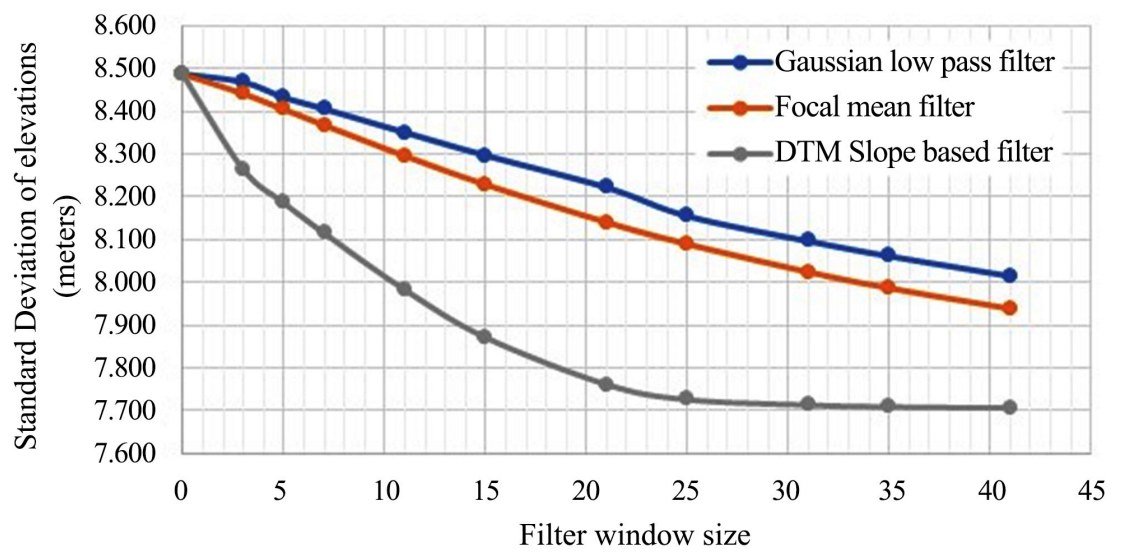

Figure 30. The relationship between the standard deviations of elevations of the created DTMs from different filtering techniques and the filter window.

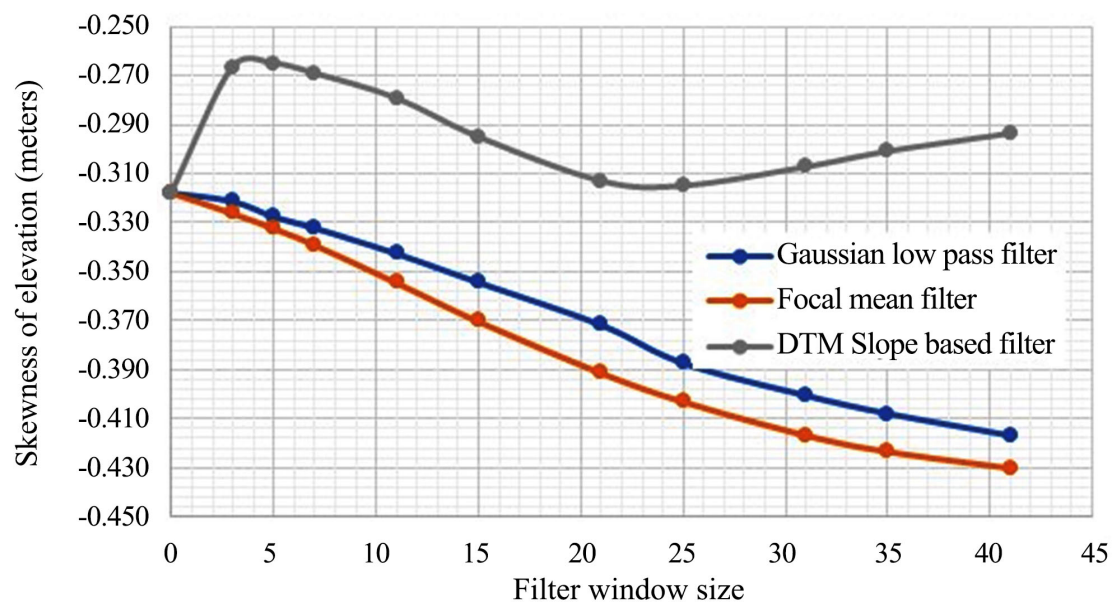

Figure 31. The relationship between the skewness of the created DTMs from different filtering techniques and the filter window size.

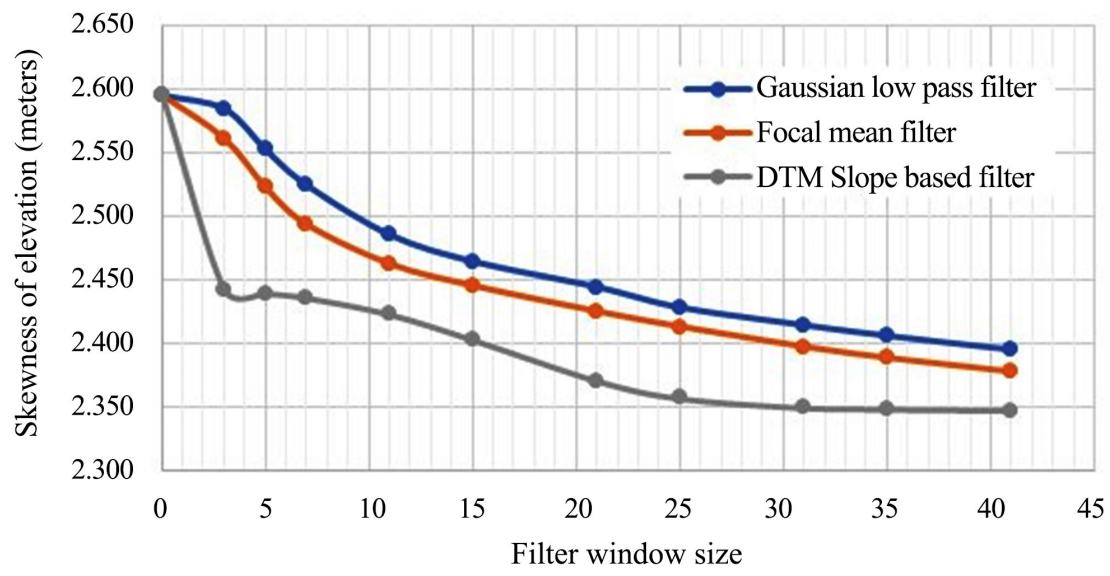

Figure 32. The relationship between the kurtosis of the created DTMs from different filtering techniques and the filter window size.

the window sizes of the different filtering approaches. Such decreases in the maximum elevations of the produced DTMs from Gaussian low pass filter and 
those from the focal analysis mean filter due to increases in the window sizes of the filters have been almost steady while the corresponding decreases in the maximum elevation have been irregular in the case of the bare earth models produced from the DTM slope-based filter. The composite behaviors of the minimum elevations and the maximum elevations have been reflected in Figure 29 which is a chart that depicts the relationship between the ranges of elevations of the created DTMs from the three filtering approaches against the filter window sizes since the ranges of elevations in a specific DTM is calculated as the difference between the maximum elevation minimum elevation in that DTM. In Figure 29 the ranges of elevations in the DTMs from Gaussian low pass filter and those from the focal analysis mean filter have decreased with increasing the filter window sizes till $31 \times 31$ where they nearly have kept nearly unchanged with larger window sizes. However, the ranges of elevations in the bare land models from the DTM slope-based filter have decreased with smaller rates due to increases in the window sizes of the filter till $35 \times 35$ where they have become unchanged with more increases in the window sizes. The situation is a bit different when studying the standard deviation of the extracted DTMs from different filtering techniques against the window size of the filter since the standard deviations of the created DTMs have decreased with increasing the window sizes of the three filters as presented in Figure 30. Thus, the decreases of the standard deviations have been continuous and steady in the DTMs from Gaussian low pass filter and those from the focal analysis mean filter while in the case of bare earth models produced from the DTM slope-based filter the decreases have occurred with high rates till a filter window size of $31 \times 31$. However, more increases in the window size of the DTM slope-based did not leave noticeable changes on the standard deviation of the produced bare earth models. Figure 31 depicts the relationship between the skewness of the created DTMs from different filtering techniques and the filter window sizes. Increases in the window size of the Gaussian low pass filter and the focal analysis mean filter have produced DTMs of less skewness and more symmetrical Gaussian curves. This has not been always the case in the bare earth models from the DTM slope-based filter where increasing of the window size of the DTM slope-based filter has produced bare earth models of fluctuated skewness values. However, the DTMs produced from Gaussian low pass filter and from focal analysis mean filter in addition to the bare earth models obtained from the DTM slope-based filter have recorded decreases in the kurtosis due to increases in the window sizes of the filters as shown in Figure 32. This means that application of these filters with increases in the window sizes has produced better removal of noise and outliers giving more consistent DTMs and bare earth models.

\section{Conclusions}

Three filtering approaches for stripping off above ground objects namely; Gaussian low pass filter, focal analysis mean filter and DTM slope-based filter at va- 
rying window sizes have been applied on airborne LiDAR DSM for creation of a reliable DTM since a DTM can be involved a wide range of environmental and engineering applications. A dataset of airborne LiDAR data of the ISPRS WG III/4 Test Project on Urban Classification and 3D Building Reconstruction that was captured over Vaihingen in Germany over a pure residential area with small detached houses on 21 August 2008 by Leica Geosystems has been used in the study. Visual analysis has indicated that Gaussian low pass filter with varying window sizes has produced blurred DTMs which has been indications of attenuation of high frequencies that refer to non-ground objects with emphasizing of low frequencies pointing to the ground surface points. With increasing the window size of the Gaussian low pass filter much blurrier DTMs have been obtained referring to better attenuation of high frequencies and consequently better removal of non-ground objects. This has been clarified by the outcomes of the statistical analysis of the DTMs from the Gaussian low pass filters which has referred to decreases in the minimum elevations, maximum elevation, ranges of elevations, standard deviations of the DTMs in addition to skewness and kurtosis of DTMs, due to increasing the window sizes of the Gaussian low pass filter resulting in attenuation of increasing amounts of the high frequencies and emphasizing of the DSM low frequencies. On the other hand, visual analysis of the DTMs created from the application of the focal analysis mean filter has shown better removal of the above ground objects compared to that from Gaussian low pass filter especially at large window sizes. Thus, details of the non-ground objects have been almost diminished in the DTMs produced from the focal analysis filter of window sizes of $25 \times 25,35 \times 35$ and $41 \times 41$ although the statistical analysis results of the DTMs from focal analysis mean filter resemble to great extent those of the DTMs from Gaussian low pass filter of varying window sizes. Visual analysis of the bare earth models extracted through the use of the DTM slope-based filter has shown very different results compared to those from the other two filters. The bare earth models obtained from the application of the DTM slope-based filter have been full of gabs of no data values at the positions of the removed non-ground objects. The sizes and numbers of the no-data gabs have increased with increasing the window sizes of the DTM slope-based filter. With the application of the tool; "close the gap with spline" working under SAGA GIS software clear views pointing to bare earth models that can be DTMs have been obtained referring to efficient removal of non-ground objects from the LiDAR DSM especially at large window sizes of the filter.

Comparative analysis of the three filters together has shown that the minimum elevation of the DTMs from Gaussian low pass filter and those from the focal analysis mean filter have increased with increasing the widow sizes of the filter till window sizes of $21 \times 21$ and $31 \times 31$ in the case of Gaussian low pass filter and focal analysis mean filter respectively. On the other hand, the DTM slope-based filtering approach has kept the minimum elevation of the original DSM unchanged. However, the three filtering approaches have produced DTMs 
of decreasing maximum elevations due to increases in the filter window sizes. Also, the standard deviations of the created DTMs have decreased with increasing the window sizes of the three filters however, the decreases have been continuous and steady in the cases of Gaussian low pass and the focal analysis mean filters. This has not been the case for the bare earth models created from DTM slope-based filter where decreases in the standard deviations have been at high rates till window sizes of $31 \times 31$ in the mean while the standard deviation has kept unchanged with more increases in the filter window sizes. Also, increases in the window size of the Gaussian low pass filter and the focal analysis mean filter have produced DTMs of decreasing skewness and more symmetrical Gaussian curves while increasing of the window size of the DTM slope-based filter has produced bare earth models of fluctuated skewness values. However, the DTMs produced from Gaussian low pass filter and from focal analysis mean filter in addition to the bare earth models obtained from the DTM slope-based filter have recorded decreases in the kurtosis due to increases in the window sizes of the filters. This means that application of these filters with increases in the window sizes has produced better removal of noise and omission of outliers giving more consistent DTMs and bare earth models. More investigation could be necessary to improve the efficiency of the three filtering approaches in removal of non-ground objects and creation of reliable DTMs.

\section{Acknowledgements}

The Vaihingen data set was provided by the German Society for Photogrammetry, Remote Sensing and Geoinformation (DGPF) [Cramer, 2010]:

http://www.ifp.uni-stuttgart.de/dgpf/DKEP-Allg.html.

\section{References}

[1] Silva, C.A., Klauberg, C., Hentz, Â.M.K., Corte, A.P.D., Ribeiro, U. and Liesenberg, V. (2018) Comparing the Performance of Ground Filtering Algorithms for Terrain Modeling in a Forest Environment Using Airborne LiDAR Data. Floresta e Ambiente, 25, e20160150. https://doi.org/10.1590/2179-8087.015016

[2] Carter, J., Schmid, K., Waters, K., Betzhold, L., Hardley, B., Mataosky, R., et al. (2012) LiDAR 101: An Introduction to LiDAR Technology, Data, and Applications. Charleston: National Oceanic and Atmospheric Administration (NOAA) Costa Services Center, Silver Spring, MD, 76p.

[3] Yunfei, B., Guoping, L., Chunxiang, C., Xiaowen, L., Hao, Z., Qisheng, H., Linyana, B. and Chaoyi, C. (2008) Classification of LiDAR Point Cloud and Generation of DTM From LiDAR Height and Intensity Data in Forested Area. The International Archives of the Photogrammetry, Remote Sensing and Spatial Information Sciences. XXXVII, 313-318.

[4] Sharma, M., Paige, G.B. and Miller, S.N. (2010) DEM Development from Ground-Based LiDAR Data: A Method to Remove Non-Surface Objects. Remote Sensing, 2, 2629-2642. https://doi.org/10.3390/rs2112629

[5] Sulaiman, N.S., Majid, Z. and Setan, H. (2010) DTM Generation from LiDAR DATA by Using Different Filters in Open-Source Software. Geoinformation Science 
Journal, 9, 1-9.

[6] Rashidi, P. and Rastiveis, H. (2018) Extraction of Ground Points from LiDAR Data Based on Slope and Progressive Window Thresholding (SPWT). Earth Observation and Geomatics Engineering, 2, 36-44.

[7] Yadav, S. (2016) Ground and Non-Ground Filtering for Airborne LIDAR Data. International Journal of Advanced Remote Sensing and GIS, 5, 1500-1506. https://doi.org/10.23953/cloud.ijarsg.41

[8] Kraus, K. and Pfeifer, N. (2001) Advanced DTM Generation from LiDAR Data. International Achieves of Photogrammetry and Remote Sensing, XXXIV-3/W4, 23-30.

[9] Priestnall, G., Jaafar, J. and Duncan, A. (2001) Extracting Urban Features from LiDAR Digital Surface Models. Computers, Environment and Urban Systems, 24, 65-78. https://doi.org/10.1016/S0198-9715(99)00047-2

[10] Wang, C.-K. and Tseng, Y.-H. (2014) Dual-Directional Profile Filter for Digital Terrain Model Generation from Airborne Laser Scanning Data. Journal of Applied Remote Sensing, 8, Article ID: 083619. https://doi.org/10.1117/1.JRS.8.083619

[11] Özcan, A.H., Ünsalan, C. and Reinartz, P. (2018) Ground Filtering and DTM Generation from DSM Data Using Probabilistic Voting and Segmentation. International Journal of Remote Sensing, 39, 2860-2883. https://doi.org/10.1080/01431161.2018.1434327

[12] Chen, C., Li, Y., Zhao, N., Guo, J. and Liu, G. (2017) A Fast and Robust Interpolation Filter for Airborne LiDAR Point Clouds. PLoS ONE, 12, e0176954. https://doi.org/10.1371/journal.pone.0176954

[13] Xing, S., Lia, P., Xu, Q., Wang, D. and Li, P. (2017) Surface Fitting Filtering of LiDAR Point Cloud with Waveform Information. ISPRS Annals of the Photogrammetry, Remote Sensing and Spatial Information Sciences, IV-2/W4, 179-184. https://doi.org/10.5194/isprs-annals-IV-2-W4-179-2017

[14] Baligh, A., Zoej, M.J.V. and Mohammadzadeh, A. (2008) Bare Earth Extraction from Airborne Lidar Data Using Different Filtering Methods. The International Archives of the Photogrammetry, Remote Sensing and Spatial Information Sciences, XXXVII, 237-240.

[15] Zhang, K. and Whitman, D. (2005) Comparison of Three Algorithms for Filtering Airborne Lidar Data. Photogrammetric Engineering \& Remote Sensing, 71, 313-324. https://doi.org/10.14358/PERS.71.3.313

[16] Chen, Z., Gao, B. and Devereux, B. (2017) State-of-the-Art: DTM Generation Using Airborne LIDAR Data. Sensors, 17, 150. https://doi.org/10.3390/s17010150

[17] Chang, Y.-C., Habib, A.F., Leeb, D.C. and Yom, J.-H. (2008) Automatic Classification of LiDAR Data into Ground and Non-Ground Points. The International Archives of the Photogrammetry, Remote Sensing and Spatial Information Sciences, XXXVII, 457-462.

[18] Hui, Z., Li, D., Jin, S., Ziggah, Y.Y., Wang, L. and Hu, Y. (2019) Automatic DTM Extraction from Airborne LiDAR Based on Expectation-Maximization. Optics \& Laser Technology, 112, 43-55. https://doi.org/10.1016/j.optlastec.2018.10.051

[19] Meng, X., Currit, N. and Zhao, K. (2010) Ground Filtering Algorithms for Airborne LiDAR Data: A Review of Critical Issues. Remote Sensing, 2, 833-860. https://doi.org/10.3390/rs2030833

[20] Hu, B., Gumerov, D., Wang, J. and Zhang, W. (2017) An Integrated Approach to Generating Accurate DTM from Airborne Full-Waveform LiDAR Data. Remote 
Sensing, 9, 871. https://doi.org/10.3390/rs9080871

[21] Liu, C., Li, J., Zhang, S. and Ding, L. (2012) A Point Clouds Filtering Algorithm Based on Grid Partition and Moving Least Squares. Procedia Engineering, 28, 476-482. https://doi.org/10.1016/j.proeng.2012.01.754

[22] Abdullah, A.F., Vojinovic, Z., Price, R.K. and Aziz, N.A.A. (2012) A Methodology for Processing Raw LiDAR Data to Support Urban flood Modelling Framework. Journal of hydroinformatics, 14, 75-92. https://doi.org/10.2166/hydro.2011.089

[23] Cramer, M. (2010) The DGPF Test on Digital Aerial Camera Evaluation-Overview and Test Design. Photogrammetrie, Fernerkundung, Geoinformation, 2010, 73-82. https://doi.org/10.1127/1432-8364/2010/0041

[24] Rottensteiner, F., Sohn, G., Gerke, M. and Wegner, J.D. (2013) ISPRS Test Project on Urban Classification and 3D Building Reconstruction. ISPRS-Commission III-Photogrammetric Computer Vision and Image Analysis, Working Group III/4-3D Scene Analysis. http://www.commission3.isprs.org/wg4/.

[25] Lillesand, T.M. and Kiefer, R.W. (2000) Remote Sensing and Image Interpretation. 4th Edition, John Wiley \& Sons, Inc., Hoboken, NJ.

[26] Jensen, J. (2000) Remote Sensing of the Environment: An Earth Resource Perspective. Pearson Prentice Hall, Upper Saddle River, NJ.

[27] Jensen, J. (2005) Introductory Digital Image Processing-A Remote Sensing Perspective. 3rd Edition, Pearson Prentice Hall, Upper Saddle River, NJ.

[28] Mather, P.M. (1999) Computer Processing of Remotely-Sensed Images: An Introduction. 2nd Edition, John Wiley \& Sons Ltd., Baffins Lane, Chichester, West Sussex P019 1UD, England.

[29] Ringeler, A. (2003) Module Gaussian Filter. SAGA-GIS Module Library Documentation (v2.2.5).

[30] Abdalla, A. and Elmahal, A.-El. (2015) Augmentation of Vertical Accuracy of Digital Elevation Models Using Gaussian Linear Convolution Filter. 2016 Conference of Basic Sciences and Engineering Studies (SGCAC), Khartoum, Sudan, 20-23 February 2016. https://doi.org/10.1109/SGCAC.2016.7458031

[31] Vosselman, G. (2000) Slope Based Filtering of Laser Altimetry Data. IAPRS, XXXIII, 935-942.

[32] Wichmann, V. (2010) Module DTM Filter (Slope-Based). SAGA-GIS Module Library Documentation (v2.2.5). http://www.saga-gis.org/saga_tool_doc/2.2.5/grid_filter_7.html

[33] Sithole, G. (2001) Filtering of Laser Altimetry Data Using a Slope Adaptive Filter. International Achieves of Photogrammetry and Remote Sensing, XXXIV-3/W4, 203-210.

[34] Sithole, G. and Vosselman, G. (2003) Report: ISPRS Comparison of Filters. ISPRS Commission III, Working Group 3 \& Department of Geodesy, Faculty of Civil Engineering and Geosciences Delft University of Technology the Netherlands. 